\title{
Burden of Illness in Type 2 Diabetes Mellitus
}

\author{
Anthony Cannon, MD, FACE; Yehuda Handelsman, MD, FACP, FNLA, FASPC, MACE;
} Michael Heile, MD, FAAFP; and Michael Shannon, MD

\begin{abstract}
An estimated 30.2 million Americans have diabetes, and this number is expected to increase based on trends over recent decades and compounded by an aging U.S. population. As reviewed in this article, type 2 diabetes mellitus (T2DM) is associated with impaired health-related quality of life (HRQoL) and with a substantial socioeconomic burden. Compared with individuals without T2DM, those with T2DM have worse HRQoL, greater decrements in HRQoL over time, and possibly greater depressive symptomology. Diabetes-related complications and comorbidities (e.g., obesity and cardiovascular disease) are associated with worse HRQoL. Hypoglycemic episodes are associated with reduced HRQoL and greater levels of depression; they can also interfere with social and occupational activities. In turn, low HRQoL can be a driver for poor glycemic control. In 2012, the total estimated cost associated with diagnosed diabetes in the United States was $\$ 245$ billion. Factors contributing to increased health care resource utilization and costs in patients with T2DM include medical comorbidities, diabetes-related complications, inadequate glycemic control, and hypoglycemic episodes. Readmission is a key driver of hospital-related costs and is more common among elderly patients with T2DM. Elderly patients with T2DM represent a particularly vulnerable population given that these patients may have varying degrees of physical and mental comorbidities that can increase their risk of hypoglycemia, falls, and depression.

This review demonstrates that T2DM imposes a considerable burden on both the individual and society. Treatment strategies should consider the effects of treatment on HRQoL and on outcomes (e.g., complications and hypoglycemia) that affect both $\mathrm{HRQ} \mathrm{OL}$ and costs. Management strategies that maximize $\mathrm{HRQ}$ oL while minimizing the risk of hypoglycemia and other treatment-related complications are particularly critical in the elderly.
\end{abstract}

J Manag Care Spec Pharm. 2018;24(9-a):S5-S13

Copyright $\odot 2018$, Academy of Managed Care Pharmacy. All rights reserved.

A n estimated 30.3 million Americans have diabetes (9.4\% of the population). Of these, 23 million people have diagnosed diabetes and 7.2 million people have undiagnosed disease. ${ }^{1}$ The prevalence of diabetes in the U.S. population has increased dramatically over the past half century (Figure 1). ${ }^{2}$ Approximately $40 \%$ of Americans with diabetes are aged 65 years or older, and $25 \%$ of all U.S. adults over age 65 have diabetes. ${ }^{1}$ As the U.S. population ages, the number of patients with diabetes is expected to increase; by 2030, the number of people in the United States with diagnosed or undiagnosed diabetes is projected to increase to 55 million. ${ }^{3}$

Type 2 diabetes mellitus (T2DM) accounts for 90\%-95\% of diabetes cases and is characterized by relative insulin deficiency, peripheral insulin resistance, and high blood glucose levels. ${ }^{4,5}$ In patients with T2DM, insulin secretion is insufficient to compensate for insulin resistance. Risk factors for T2DM include older age, obesity, family history of diabetes, a history of gestational diabetes, physical inactivity, impaired glucose metabolism, and race/ethnicity, with African Americans, Hispanics/Latinos, Native Americans, Asian populations (especially Indians and Filipinos), and Native Hawaiians or other Pacific Islanders being at an increased risk. ${ }^{1,4}$ Globally, the prevalence of diabetes (percentage of population) in adults aged 20-79 years is highest in Central America and the Middle East/northern Africa. ${ }^{6}$

\section{Diabetes-Related Complications}

Uncontrolled diabetes is associated with the development of complications that may compromise health-related quality of life (HRQoL) and may increase mortality risk. These include macrovascular (e.g., coronary artery disease, stroke, and peripheral vascular disease) and microvascular (e.g., retinopathy, nephropathy, and neuropathy) complications. ${ }^{7}$ Global data from diverse independent studies have reported increased relative risk of stroke ranging from 1.4 to 5.8 in people with diabetes. ${ }^{8}$ After adjustment for population age differences, cardiovascular disease death rates in 2003-2006 were about 1.7 times higher among adults aged 18 and older with diagnosed diabetes than among those without diabetes. ${ }^{4}$

During 2011-2012, the estimated crude prevalence of chronic kidney disease (stages 1-4) was 36.5\% among U.S. adults aged 20 years and older with diagnosed diabetes. ${ }^{9}$ Diabetes is a key cause of end-stage renal disease and, in 2011, was listed as the primary cause of renal failure in $44 \%$ of new cases. ${ }^{4}$ In 2011, nearly 50,000 people initiated treatment for renal failure due to diabetes, and 228,924 people with renal failure due to diabetes were receiving chronic dialysis or living with a kidney transplant. ${ }^{4}$ In 2014 , more than 52,000 U.S adults developed end-stage renal disease with diabetes as the primary cause. $^{10}$

Diabetes complications such as peripheral neuropathy and retinopathy lead to potentially disabling sequelae. Diabetes accounts for approximately $60 \%$ of non-traumatic lower-limb amputations among people aged 20 and older. ${ }^{4}$ Retinopathy affects an estimated 28.5\% of adults with diabetes aged 40 years and older. ${ }^{4}$ About $4 \%$ of adults aged $\geq 40$ years with diabetes have advanced diabetic retinopathy, with associated conditions (e.g., macular edema and proliferative diabetic retinopathy) that result in severe vision loss. Notably, diabetic retinopathy is the leading cause of blindness among U.S. adults. ${ }^{11}$

This review provides an overview of the burden of T2DM, including the effects on HRQoL and economic impact, with a focus on the U.S. market.

Studies Evaluating the Impact of T2DM on Quality of Life The association between diabetes and impaired HRQoL has long been recognized. ${ }^{12-14}$ The large, multinational Diabetes Attitudes, Wishes and Needs (DAWN) study evaluated psychosocial issues and experiences relating to diabetes management from the perspective of both patients and providers. ${ }^{15}$ Among 


\section{FIGURE 1 Number and Percentage of U.S. Population with Diagnosed Diabetes, 1958-2015²}

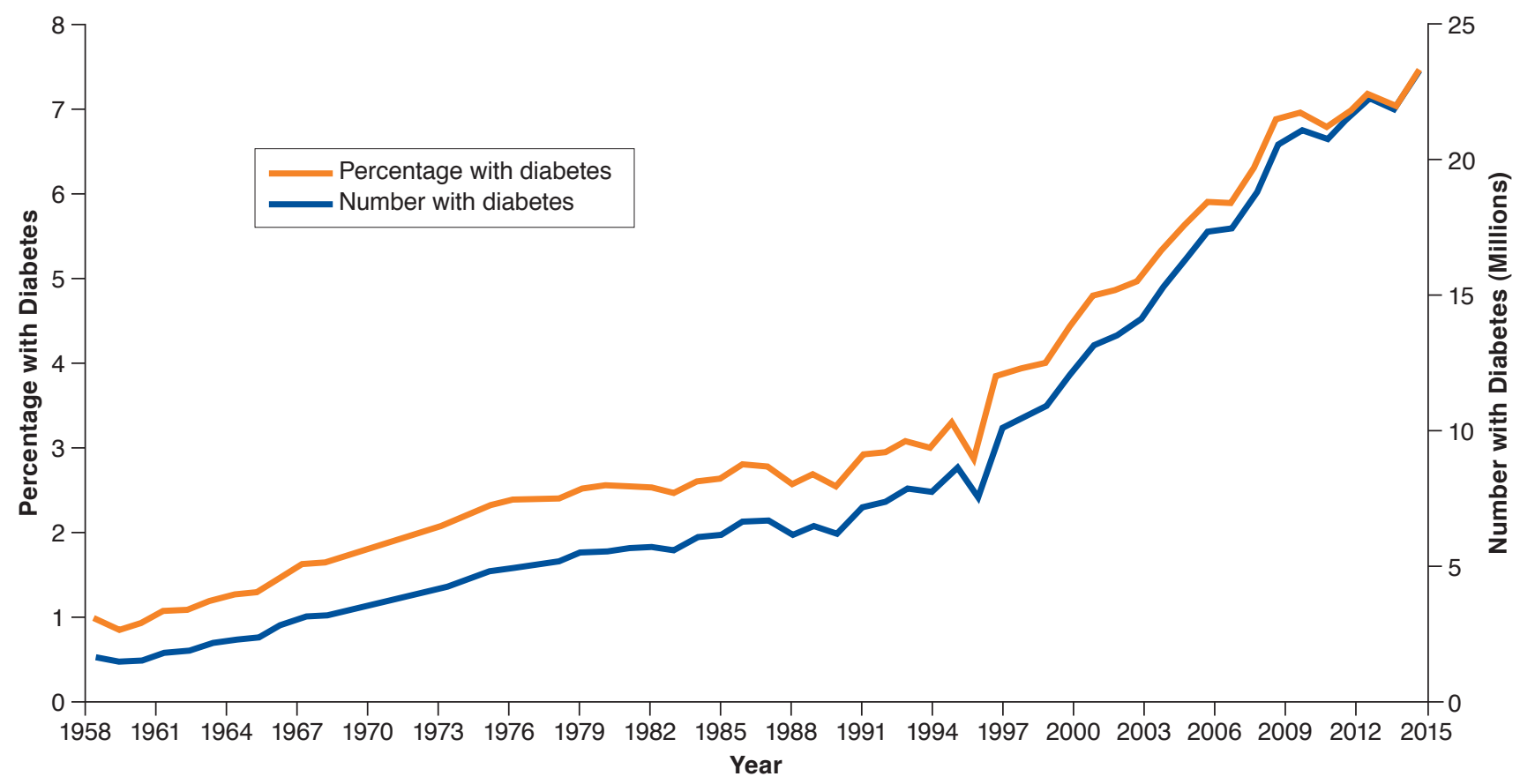

From Centers for Disease Control and Prevention, Division of Diabetes Translation. United States Diabetes Surveillance System, available at: http://www.cdc.gov/diabetes/data.

those with T2DM, $41 \%$ of patients reported that they experienced poor psychological well-being. Additionally, providers (including nurses, primary care physicians, and specialists) estimated that $62 \%-72 \%$ of their patients with T2DM had psychological problems, including depression, anxiety, stress, and burnout.

The Study to Help Improve Early evaluation and management of risk factors Leading to Diabetes (SHIELD) was a 5-year, U.S.-based survey study initiated in 2004 that was designed to enhance understanding of patterns of attitudes, knowledge, and health behavior of individuals with T2DM and those with varying levels of cardiometabolic risk. ${ }^{16}$ This study included the Short-Form 12-item Health Survey (SF-12), a brief measure of overall health status, and the 9-item Patient Health Questionnaire (PHQ-9), an assessment of depression. The SF-12 was completed by adults across the United States with T2DM $(n=3,530)$ and by those determined to be at high risk $(n=5,051)$ or low risk $(n=5,335)$ for T2DM based on the presence of recognized risk factors. Compared with low-risk respondents, those with T2DM were found to have significantly reduced physical, but not mental, HRQoL (Figure 2) and also had greater depressive symptomology and higher rates of moderate to severe depression (19.6\% vs. $11.3 \%$ ).
The SHIELD study also longitudinally assessed changes in health status and HRQoL in patients with T2DM using the EuroQol-5D (EQ-5D) instrument for measuring quality of life. This was completed at baseline and 5 years later by 1,741 patients with T2DM and 4,543 respondents without diabetes. ${ }^{17}$ Patients with T2DM had impaired HRQoL compared with those without diabetes at baseline and at 5 years $(P<0.0001$ for both index and visual analog scale [VAS] scores). Among patients with T2DM followed for 5 years, HRQoL worsened over time to a significantly greater degree $(P=0.001)$ compared with adults without diabetes, indicating a long-term adverse impact on HRQoL. An international study of patients with T2DM (the Action in Diabetes and Vascular Disease: Preterax and Diamicron MR Controlled Evaluation [ADVANCE] trial) found that HRQoL among patients with T2DM declined over a 5-year follow-up period based on EQ-5D scores. ${ }^{18}$ Similarly, a German study demonstrated that patients with T2DM demonstrated large decrements in both mental and physical HRQoL over time compared with individuals without diabetes. ${ }^{19}$

\section{Impact of Diabetes-Related Complications and Comorbidities on Quality of Life}

Diabetes-related complications and comorbidities (e.g., obesity and cardiovascular disease) reduce HRQoL in patients with 


\section{FIGURE 2 Mean SF-12 Physical Component Summary and Mental Component Summary Scores in Patients} with Low Risk for T2DM, High Risk for T2DM, or T2DM in the SHIELD Study' ${ }^{16}$

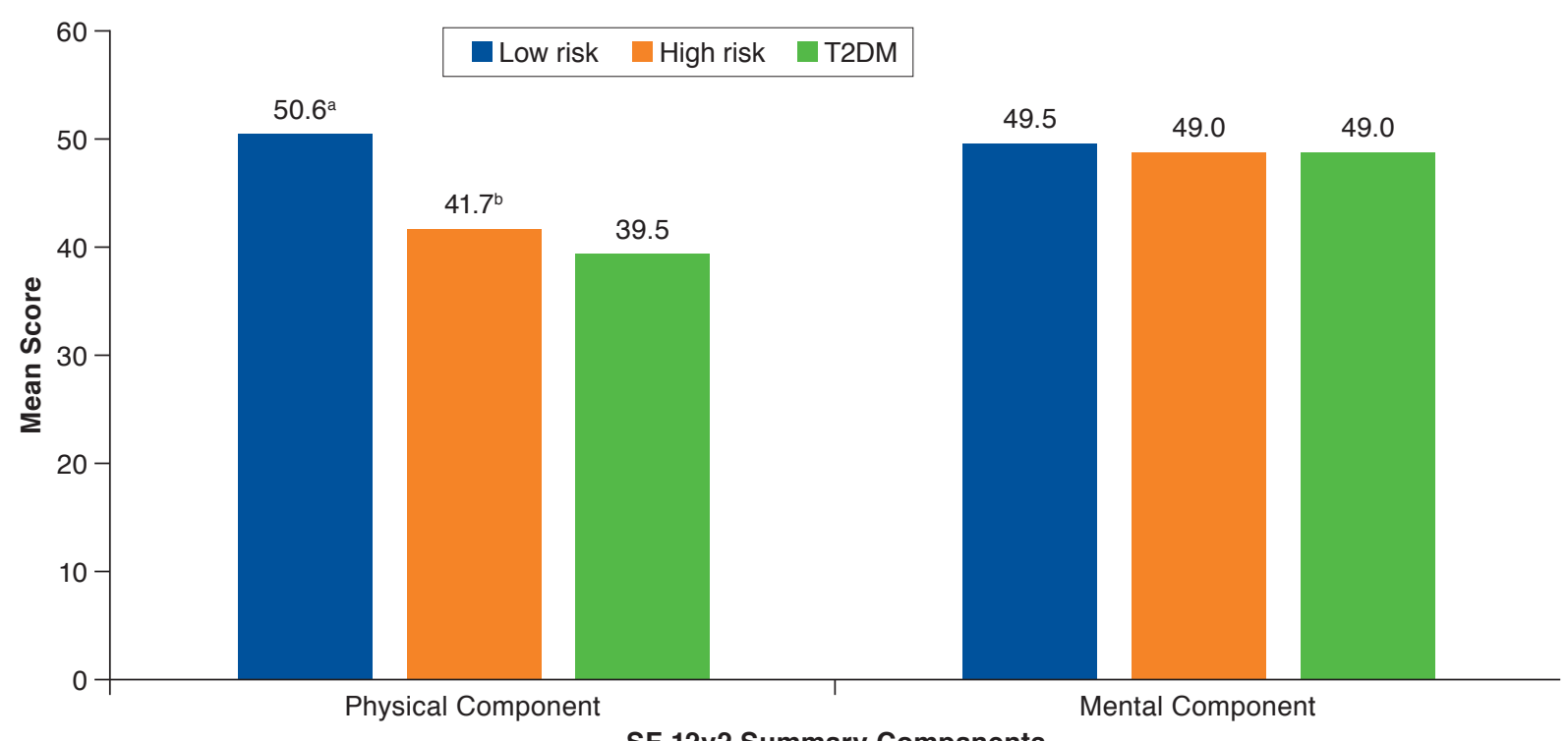

SF-12v2 Summary Companents

From Grandy S, Chapman RH, Fox KM; SHIELD Study Group. Quality of life and depression of people living with type 2 diabetes mellitus and those at low and high risk for type 2 diabetes: findings from the Study to Help Improve Early evaluation and management of risk factors Leading to Diabetes (SHIELD). Int J Clin Pract. 2008;62(4):562-68. Copyright (C) 2008. Reproduced with permission.

a $P<0.001, T 2 D M$ vs. low risk.

${ }^{b} P<0.001, T 2 D M$ vs. high risk.

SF-12v2 =Short-Form 12-item Health Survey Version 2; T2DM=type 2 diabetes mellitus .

T2DM. 20-22 The UK Prospective Diabetes Study (UKPDS) was a landmark, multicenter, randomized controlled trial of glycemic therapies in more than 5,000 patients with newly diagnosed T2DM. It demonstrated conclusively that improving blood glucose levels and/or blood pressure control reduced the risk of microvascular complications of T2DM. ${ }^{23}$ Two cross-sectional UKPDS studies evaluated the effects of intensive policies aimed at improving glycemic or blood pressure control on HRQoL. ${ }^{24}$ While these substudies did not observe an impact of the interventions on HRQoL, an important finding was that complications of T2DM were associated with impairments in HRQoL: patients with a macrovascular complication within the last year had worse general health, more problems with mobility and usual activities, and reduced vigor. Those patients with microvascular complications in the last year reported elevated levels of tension and mood disturbance.

Longitudinal assessment of the impact of diabetes complications on HRQOL in the UKPDS found that myocardial infarction (MI) caused acute/short-lived impairment of HRQoL (i.e., within 1 year of event), whereas other complications (stroke, heart failure, and amputation) resulted in longer-lasting impairment. ${ }^{25}$ Importantly, amputation, stroke, and heart failure had the greatest impact on HRQoL, whereas blindness in 1 eye and ischemic heart disease did not impact HRQoL. In the ADVANCE trial, complications of T2DM reduced HRQoL, with the greatest impact observed for amputation, followed by stroke, blindness, renal failure, heart failure, and MI. ${ }^{18}$

\section{Secondary Quality of Life Impact from Diabetes Treatment}

Diabetes treatments can pose risks of dangerously low blood glucose levels, or hypoglycemic events, which are particularly feared by patients and can negatively impact HRQoL. ${ }^{26,27}$ Patients with T2DM worry about passing out in public or injuring themselves due to a hypoglycemic episode..$^{28}$ In the SHIELD study, respondents with T2DM reporting at least 1 hypoglycemic episode in the past 12 months had significantly worse physical and mental health on the SF-12 than respondents without hypoglycemia; mean PHQ-9 scores were also higher among those with hypoglycemia, indicating greater levels of depression. ${ }^{29}$ Similar results were observed when comparing respondents with hypoglycemia versus those without hypoglycemia over the past 4 weeks. In addition, among respondents reporting hypoglycemia over the past 4 weeks, mean SF-12 physical component summary scale scores decreased, and 
mean PHQ-9 scores increased as the number of reported hypoglycemic episodes increased.

The U.S. National Health and Wellness Survey (NHWS), a large internet-based study of adults aged $\geq 18$ years, found that hypoglycemia negatively impacted HRQoL in patients with T2DM and, in addition to interfering with social activities, was associated with absenteeism (missed work), presenteeism (impairment while at work), and decreased overall work productivity. ${ }^{30}$ Further, a European survey of 5,817 patients aged 40 years and older with T2DM duration of at least 1 year found that patients experiencing any hypoglycemic episode over the past year reported greater diabetes-related impairment on multiple measures of HRQOL and health status compared with those with no hypoglycemia episodes. ${ }^{27}$

Hypoglycemia in T2DM patients treated with oral antihyperglycemic drugs is associated with impaired HRQoL, with increases in severity and frequency of hypoglycemia associated with greater decreases in HRQoL. ${ }^{31,32}$ In a European multicenter observational study (the Real-Life Effectiveness and Care Pattern of Diabetes Management study) that enrolled patients with T2DM on oral antihyperglycemic agents metformin plus a sulfonylurea or thiazolidinedione, 38\% of 1,709 patients reported hypoglycemic symptoms in the 12 months before enrollment. Of patients with hypoglycemic symptoms, the most severe symptoms were mild for $68 \%$ of patients, moderate for $27 \%$, and severe for $5 \%$. Adjusted linear regression analyses indicated that patients reporting hypoglycemic symptoms had significantly worse HRQoL (based on EQ-5D VAS) scores and that impairment in HRQoL increased with greater hypoglycemic symptom severity. Similarly, a follow-up survey (the 2007 U.S. NHWS) of patients with self-reported T2DM treated with oral antihyperglycemic drugs (with half of patients taking a treatment regimen that included a sulfonylurea) found that $63 \%$ of patients reported hypoglycemic episodes (46\% mild, $37 \%$ moderate, $13 \%$ severe, and $4 \%$ very severe) and that patients reporting any hypoglycemic symptoms had significantly worse HRQoL based on EQ-5D utility scores than patients without hypoglycemic symptoms. ${ }^{32}$ Adjusted linear regression analysis revealed that decrements in HRQoL (based on EQ-5D utility scores) increased with increasing severity of hypoglycemic episodes.

Another potential treatment-related burden pertains to changes in body weight. Obesity is a common comorbidity of diabetes, and obesity contributes independently to lower HRQoL. ${ }^{21,33}$ Weight gain secondary to diabetes treatment can potentially add to the HRQoL burden of the disease. A number of diabetes treatments are well known for their potential to cause weight gain, including insulin, sulfonylureas, and thiazolidinediones. ${ }^{34}$

\section{Clinical Consequences of Quality of Life Burdens}

The phenomenon of "diabetes-related distress" refers to the negative emotional reactions to a diabetes diagnosis, demands of self-management, risk of complications, inadequate provider care, and unsupportive interpersonal relationships. ${ }^{35}$ One author has termed this phenomenon "diabetes overwhelmus." 36 Ironically, poor HRQoL can, in turn, be a driver leading to poor glycemic control, which can increase risks for greater comorbidity and added HRQoL burden, thus creating a vicious cycle. $^{37}$ Evaluating diabetes-related distress and addressing underlying factors may potentially improve patients' HRQoL and self-care capacity.

Another potential contributor to diabetes-related distress is the logistical characteristics of diabetes management, such as the pill/injection burden, along with the glucose testing burden. Patients with diabetes often feel overwhelmed and frustrated by the daily hassles of disease management. ${ }^{38}$ Such feelings may contribute not just to reduced HRQoL, but could have broad negative impacts on diabetes self-management, possibly leading to poor clinical outcomes.

\section{The Economic Impact of Diabetes}

In 2012, the total estimated cost of diagnosed diabetes in the United States was $\$ 245$ billion, comprising $\$ 176$ billion in direct medical costs and $\$ 69$ billion in lost productivity due to work-related absenteeism, reduced productivity at work and at home, unemployment attributable to chronic disability, and premature mortality. ${ }^{39}$ Hospital inpatient care accounted for $43 \%$ of total medical costs. Diabetes-related costs in the United States are projected to reach $\$ 622.3$ billion in 2030 , including $\$ 472$ billion in annual medical costs. ${ }^{3}$ The estimated additional lifetime cost of treating T2DM and associated complications in U.S. patients differs based on gender and age at diagnosis. ${ }^{40}$ For men, lifetime costs range from $\$ 54,700$ for those diagnosed at age $\geq 65$ years to $\$ 124,700$ for those diagnosed between age 25 and 44 years. For women, lifetime costs range from $\$ 56,600$ for patients diagnosed at age $\geq 65$ years to $\$ 130,800$ for those diagnosed between age 25 and 44 years.

In California, about one third of hospitalizations in patients aged 35 years and older occur in people with diabetes. ${ }^{41}$ Regardless of primary diagnosis, the average hospital stay costs almost $\$ 2,200$ more for a patient with diabetes versus a patient without diabetes $(\$ 18,691$ vs. $\$ 16,492)$. A retrospective study examined health care resource utilization (HCRU) and costs for patients with T2DM and matched case controls in a Medicare Advantage Prescription Drug plan. ${ }^{42}$ Compared with controls without T2DM, patients with T2DM had significantly higher mean annual all-cause health care costs per patient associated with inpatient hospitalizations; outpatient, office, and emergency department (ED) visits; pharmacy expenditures; and total health care costs. The excess total annual cost for patients with T2DM was $\$ 3,120$, and the excess total medical cost was $\$ 2,689$. Patients with T2DM also had significantly greater HCRU with respect to inpatient hospitalization, average number of inpatient days, outpatient visits, office visits, ED visits, and pharmacy claims. 
Managed care administrative claims data were used to identify high-cost patients with T2DM, defined as those patients with costs in the top $10 \%$ of the overall cost distribution. ${ }^{43}$ Annual per-patient costs were $\$ 56,468$ for high-cost patients compared with $\$ 4,674$ for non-high-cost patients. Logistic regression was used to assess predictors of being a high-cost T2DM patient. High underlying comorbidity burden (Charlson Comorbidity Index $\geq 2$ ) was the strongest predictor of being a high-cost patient (odds ratio $[\mathrm{OR}]=4.896$ ), followed by renal impairment $(\mathrm{OR}=2.368)$, obesity $(\mathrm{OR}=2.106)$, and insulin use $(\mathrm{OR}=2.098)$.

Readmission is a key driver of costs in hospitalized patients with T2DM. In a retrospective cohort analysis of data from patients with T2DM enrolled in Humana Medicare Advantage with Prescription Drug Insurance and commercial insurance plans, mean 12-month all-cause medical costs were highest for patients with an inpatient admission and 30-day readmission $(\$ 76,806)$, followed by patients with an inpatient admission without a 30 -day readmission $(\$ 42,011)$, and lowest for matched patients without an inpatient admission $(\$ 9,624$; $P<0.001) .{ }^{44}$ Similar patterns were observed for all-cause medical costs, all-cause pharmacy costs, DM-related total costs, DM-related medical costs, and DM-related pharmacy costs. Notably, the risk of 30-day hospital readmission is significantly higher among individuals aged 75 years and older with T2DM relative to patients younger than 65 years of age. ${ }^{45}$ Ravel et al. (2015) examined all-cause 30-day hospital readmissions among Medicare beneficiaries aged $\geq 65$ years with T2DM. ${ }^{46}$ Overall, 13.2\% of hospitalized patients were readmitted within 30 days of initial hospitalization. Age $\geq 75$ years was associated with a greater risk of readmission, as was cognitive impairment, depression, falls and fall risk, polypharmacy, and urinary incontinence; depression was not significant in multivariate analysis.

Diabetes-related complications contribute to the economic burden of T2DM. Approximately 20\% of commercial insurance or Medicare costs in patients with T2DM are attributable to diabetes-related complications. ${ }^{47}$ Among Medicare beneficiaries with T2DM, an increase in complications burden (representing the number and severity of complications) was associated with increased total costs. ${ }^{48}$ Every 1-point increase in complications burden on the Diabetes Complications Severity Index was associated with a $\$ 2,744$ increase in total costs over 24 months, including a $\$ 2,480$ increase in medical costs and a $\$ 264$ increase in pharmacy costs.

An analysis of data from the MarketScan Commercial Claims and Encounters Database estimated HCRU and costs per episode of care for complications of T2DM.49 More than 34.7 million episodes of care were identified among 1,846,287 patients. The mean adjusted costs per episode were $\$ 16,435$ for coronary artery disease (CAD) with acute MI, ventricular fibrillation, shock, and/or cardiac arrest; $\$ 4,558$ for cerebrovascular disease (CVD) with stroke; $\$ 445$ for hypoglycemia; $\$ 5,675$ for complications of diabetes (including cellulitis, pyelonephritis, gangrenous infection, osteomyelitis, hyperosmolar state, ketoacidosis, acute MI, acute cerebrovascular accident, sepsis, coma, hyperosmolar coma, and shock); and $\$ 8,765$ for renal failure (Figure 3). For each category of care (e.g., inpatient and ED), the cost was determined for the patients who had at least 1 visit related to that category of care (i.e., cost of inpatient care among patients with inpatient care, cost of ED visits among patients with at least 1 ED visit). Inpatient visits represented the largest component of costs for CAD, CVD, and complication episodes, whereas other ancillary care accounted for the largest cost component for hypoglycemia and renal failure.

Ward et al. (2014) estimated the direct medical costs per patient for complications of T2DM, including costs of managing the acute episode and subsequent care within the first year following the event. ${ }^{50}$ Highest costs were observed for endstage renal disease $(\$ 71,714)$, acute MI $(\$ 56,445)$, and ischemic stroke $(\$ 42,119)$. Based on an analysis of U.S. administrative claims data, total initial cost for MI ranged from $\$ 28,014$ for Medicare to $\$ 41,505$ for Medicaid, and total cost for followup care within 1 year ranged from $\$ 2,036$ for commercial insurance to $\$ 4,799$ for Medicaid ${ }^{51}$; for stroke, total initial cost ranged from $\$ 22,657$ for Medicare to $\$ 40,370$ for Medicaid, and total cost for follow-up care within 1 year ranged from $\$ 2,488$ for Medicare to $\$ 4,584$ for Medicaid.

Inadequate glycemic control may contribute to the economic burden of T2DM. Achieving and sustaining glycemic targets is recommended to reduce the risk of complications in patients with T2DM.,52 Despite the broad spectrum of treatment options for T2DM, many patients still have poor glycemic control. One estimate found that, in 2012, about 7.4 million (57\%) insured adults with T2DM had controlled diabetes, and 5.6 million (43\%) had uncontrolled diabetes based on International Classification of Diseases, Ninth Revision, Clinical Modification (ICD-9-CM) diagnosis codes (any ICD-9-CM code indicating uncontrolled diabetes during the year). ${ }^{53}$ In another analysis, the percentage of patients represented in a database of privately insured and Medicare Advantage patients with uncontrolled T2DM (defined as Alc $\geq 9 \%$ ) increased from 9.9\% in 2006 to $12.2 \%$ in $2013 .{ }^{54}$

A retrospective database analysis of medical and pharmacy claims and laboratory data from a large U.S. managed care health plan reported that, after controlling for confounding factors, the predicted total diabetes-related costs over a 1-year follow-up period were 32\% higher for patients with T2DM whose Alc was above $7 \%$ relative to patients with Alc $\leq 7 \%$ ( $\$ 1,540$ vs. $\$ 1,171$ per patient; $P<0.001) .{ }^{55}$ Multivariate analysis of medical and pharmacy administrative claims data from a large managed care organization showed that Alc correlated significantly with diabetes-related medical costs for patients with T2DM, with each $1 \%$ increase in Alc corresponding to a 


\section{FIGURE 3 Adjusted Health Care Costs per Episode by Episode Type, Patients with Utilization in Each} Care Settinga

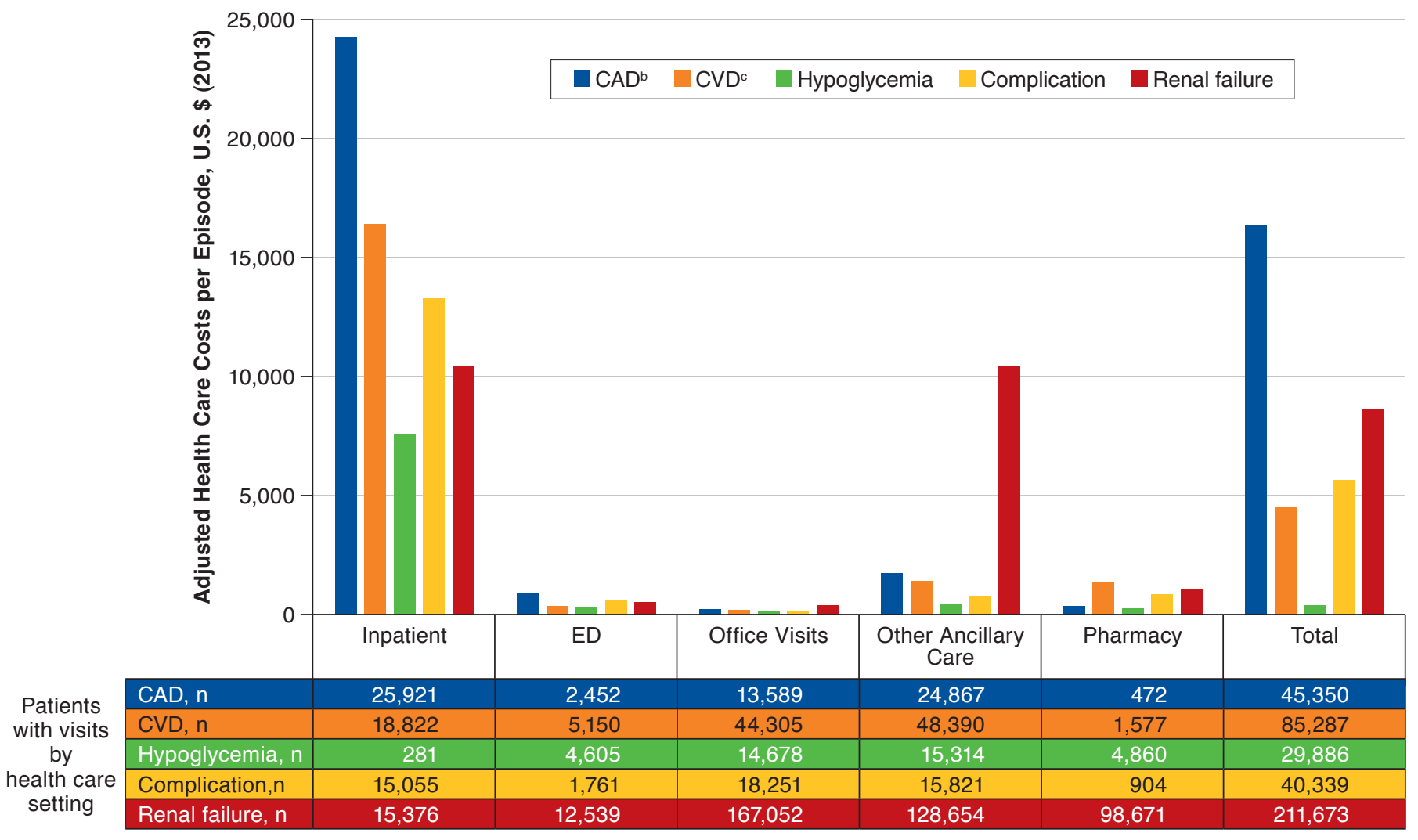

a Data from Candrilli et al. (2015). ${ }^{49}$

${ }^{b} C A D$ episodes include coronary artery disease with acute myocardial infarction, ventricular fibrillation, shock, and/or cardiac arrest.

${ }^{C} \mathrm{CVD}$ episodes include cerebrovascular disease with stroke.

$C A D=$ coronary artery disease $C V D=$ cerebrovascular disease; $E D=$ emergency department.

4.4\% increase in diabetes-related medical costs (translating to an annual cost increase of $\$ 250$ per year). ${ }^{56}$

Costs associated with hypoglycemia are considerable. A literature review that combined T1DM and T2DM concluded that both severe and nonsevere hypoglycemia episodes were associated with substantial costs related to HCRU and lost productivity. ${ }^{57}$ For older adults with T2DM obtaining tight glycemic control (Alc $<6.5 \%$ ) with insulin or sulfonylureas, the annual costs of hypoglycemia in the United States exceed \$509 million. ${ }^{58}$ Notably, retrospective analysis of a pharmacy administrative claims database found that age- and sex-standardized rates of severe hypoglycemia resulting in hospital admission, observation stay, or ED visit did not improve between 2006 and 2013.54

Interestingly, the economic impact of $\mathrm{T} 2 \mathrm{DM}$ is a further contributor to the overall HRQoL burden. As medical expenditures associated with diabetes increase, HRQOL in patients with diabetes decreases. ${ }^{59}$ Campbell et al. (2017) used data from the 2002-2011 Medical Expenditure Panel Survey Household Component to examine the relationship between medical expenditures and HRQoL among U.S. adults with diabetes (type not specified). ${ }^{59}$ Quality of life (QoL) was measured using the Physical Component Summary (PCS) and Mental Component Summary (MCS) derived from the SF-12v2, and scores were converted into quartiles, with lower quartiles reflecting worse QoL. After adjusting for covariates (including, among others, age, sex, health insurance status, and comorbidities), increasing mental and physical HRQoL were associated with both lower total expenditures and lower out-of-pocket costs.

\section{Considerations in Older Patients}

The prevalence of diabetes and complications is increasing in older patients, leading to increasing burdens related to medical 
care and HRQoL impairment. ${ }^{60}$ In 2012, individuals aged 65 years and older accounted for 59\% of U.S. health care expenditures attributable to diabetes, and diabetes-related nursing/ residential care costs among those aged $\geq 65$ years were approximately $\$ 11.8$ billion. ${ }^{39}$ T2DM affects $6.1 \%$ of commercially insured individuals aged between 20 and 64 years and 19.4\% of Medicare beneficiaries aged 65 years and older. ${ }^{47}$ Diabetes is particularly problematic in the long-term care (LTC) setting: The estimated prevalence of diabetes is $32.8 \%$ among elderly nursing home residents. ${ }^{61}$

In older patients with diabetes, cognitive impairment and dementia may further compromise HRQoL. ${ }^{62}$ A survey of U.S. adults found that older age, especially aged $\geq 75$ years, was associated with a greater risk of self-rated fair or poor health. ${ }^{14}$ In the ADVANCE trial, described above, greater decrements in HRQOL over time were observed with increasing age among patients with diabetes. ${ }^{18}$

Older patients may have varying degrees of physical and mental comorbidities, such as cognitive impairment, chronic pain, and renal impairment, that can increase their risk of hypoglycemia, falls, and depression. These comorbidities, and the polypharmacy that is often used to treat multiple conditions, can lead to impairment of glycemic control and HRQoL. ${ }^{63}$ In particular, older patients are at an increased risk of hypoglycemia, which may lead to falls. ${ }^{63,64}$ An analysis of a U.S. commercial database that included more than 1 million patients with T2DM demonstrated that the risk of fall-related events over 1 year increased 2 -fold among patients aged $\geq 65$ years who experienced hypoglycemia compared with patients in the same age category without hypoglycemic events. ${ }^{64}$ Older patients with hypoglycemia also had an elevated risk of fallrelated outcomes of fractures, head injuries, hospital admissions, and LTC placement.

A retrospective analysis of data from a nationally representative insurance claim database found that, among patients with T2DM, age $\geq 65$ years was a significant predictor of hospitalization (hazard ratio $=1.85$ ). ${ }^{65}$ Significant clinical predictors of hospitalization were prior diagnosis of cancer and cardiovascular, renal, arterial, and liver disease. Compared with younger patients with T2DM, those aged $\geq 65$ years also have a significantly higher proportion of hypoglycemia-related hospitalizations (38.3\% vs. 11.4\%). ${ }^{65}$ Between 1999 and 2011, rates of hospital admissions among Medicare beneficiaries aged $\geq 65$ years for hyperglycemia decreased, while those for hypoglycemia increased; among older adults, hospital admissions for hypoglycemia now exceed those for hyperglycemia. ${ }^{62}$ As described above, hospitalized patients with T2DM aged 75 years and older have an elevated risk of 30-day hospital readmission. $.55,46$

According to the American Diabetes Association (ADA), overall health, medical comorbidities, functional status, and cognitive status should be carefully considered when setting glycemic goals and selecting a treatment regimen in older patients with T2DM. ${ }^{5}$ An Alc goal of less than $7.5 \%$ is reasonable in a healthy older adult with few comorbid illnesses and intact cognitive and functional status, whereas a less stringent target $(<8.0 \%)$ should be considered for patients with multiple comorbidities or functional or mild to moderate cognitive impairment; an Alc target of $<8.5 \%$ is reasonable for older patients with very complex/poor health. ${ }^{5}$ Risk of hypoglycemia is perhaps the most important factor in determining glycemic goals, especially in the LTC setting. ${ }^{5,66}$ As older adults tend to be on many medications, simplified treatment regimens are recommended whenever possible, and the cost of treatment should also be taken into consideration. Additional medication-specific factors that are of particular importance when managing T2DM in older patients include risk of hypoglycemia, safety in patients with renal impairment, effects on comorbidities and diabetic complications, ease/route of administration, and vigilance for drug interactions. ${ }^{66,67}$

A comprehensive approach to managing T2DM in older patients should consider the special vulnerabilities of this patient population and their clinical and functional heterogeneity. ${ }^{60}$ Such an approach should carefully evaluate the interrelationship between medical, functional, social, and psychological domains and the impact of different management strategies on these domains. For example, while a healthy older person with excellent self-management and self-efficacy skills and access to care and social support may be able to tolerate more intensive interventions, pharmacologic management becomes more challenging in the older patient as diabetes progresses, medical comorbidities develop or worsen, and factors such as functional impairment and cognitive decline limit the patient's self-management and self-efficacy capacities. It is important to review and adjust diabetes targets accordingly as patients age and to focus on goals, such as prevention of hyperglycemic emergencies, severe hypoglycemia, diabetic polyuria, and dehydration in order to preserve HRQoL. The ADA has published specific treatment guidelines for older adults. ${ }^{68}$

\section{Conclusions}

T2DM imposes a considerable burden, in particular with regard to effects on HRQoL and economic costs. Diabetes-related and treatment-related complications contribute substantially to the adverse impact of T2DM on HRQoL and health care costs. Elderly patients with T2DM are a particularly vulnerable population, and management strategies that maximize HRQoL while minimizing the risk of hypoglycemia and other treatment-related complications are warranted. Treatment strategies for T2DM should take into account the effects of treatment on HRQoL, as well as on outcomes such as microvascular and macrovascular complications and hypoglycemia that have 
pronounced effects on HRQoL and on HCRU. Recommendations to alleviate the QoL burden of patients with T2DM include selecting effective medications with lower burden of hypoglycemia, addressing complications and comorbidities that may adversely affect HRQoL, and considering factors such as cost and frequency of administration that may impact QoL.

\section{Authors}

ANTHONY CANNON, MD, FACE, Private Practice, Hamilton, New Jersey; YEHUDA HANDELSMAN, MD, FACP, FNLA, FASPC, MACE, Metabolic Institute of America, Tarzana, California; MICHAEL HEILE, MD, FAAFP, TriHealth, Cincinnati, Ohio; and MICHAEL SHANNON, MD, PMG Olympia Endocrinology, Lacey, Washington.

\section{DISCLOSURES}

This supplement was funded by Novo Nordisk. Cannon reports speaker fees and owns stock in Novo Nordisk. Handelsman reports research grants from Amgen, AstraZeneca, Bristol-Myers Squibb, Boehringer Ingelheim, Grifols, Janssen, Lexicon, Merck, Novo Nordisk, Regeneron, and Sanofi; speaker fees from Amarin, Amgen, AstraZeneca, Boehringer Ingelheim-Lilly, Janssen, Merck, Novo Nordisk, Regeneron, and Sanofi; and has served in advisory capacity to Amarin, Amgen, AstraZeneca, Boehringer Ingelheim, Eisai, Intarcia, Janssen, Lilly, Merck, Merck-Pfizer, Novo Nordisk, Regeneron, and Sanofi. Heile reports speaker fees from and has served as advisor to Novo Nordisk. Shannon reports consultant and speaker fees from Novo Nordisk and Boehringer Ingelheim-Lilly Alliance.

\section{ACKNOWLEDGMENTS}

Writing assistance was provided by Adrienne Drinkwater, PhD, and Sandra Westra, PharmD, through Churchill Communications (Maplewood, NJ) and funded by Novo Nordisk.

\section{REFERENCES}

1. Centers for Disease Control and Prevention (CDC). National diabetes statistics report, 2017. Available at: https://www.cdc.gov/diabetes/pdfs/data/ statistics/national-diabetes-statistics-report.pdf. Accessed July 5, 2018.

2. Centers for Disease Control and Prevention (CDC). Long-term trends in diabetes. April 2017. Available at: https://www.cdc.gov/diabetes/statistics/ slides/long_term_trends.pdf. Accessed July 5, 2018.

3. Rowley WR, Bezold C, Arikan Y, Byrne E, Krohe S. Diabetes 2030 insights from yesterday, today, and future trends. Popul Health Manag. 2017;20(1):6-12.

4. Centers for Disease Control and Prevention (CDC). National diabetes statistics report, 2014. Available at: https://www.cdc.gov/diabetes/pdfs/ data/2014-report-estimates-of-diabetes-and-its-burden-in-the-united-states. pdf. Accessed July 5, 2018

5. American Diabetes Association. Standards of medical care in diabetes 2018. Available at: https://professional.diabetes.org/content-page/standardsmedical-care-diabetes. Accessed July 5, 2018.

6. International Diabetes Federation. IDF Diabetes Atlas, 6th edition. Brussels: International Diabetes Federation; 2013.

7. Fowler MJ. Microvascular and macrovascular complications of diabetes. Clin Diabetes. 2008;26(2):77-82.

8. Tun NN, Anrunagirinathan G, Munshi SK, Pappachan JM. Diabetes mellitus and stroke: a clinical update. World J Diabetes. 2017;8(6):235-48.
9. Murphy D, McCulloch CE, Lin F, et al.; Centers for Disease Control and Prevention Chronic Kidney Disease Surveillance Team. Trends in prevalence of chronic kidney disease in the United States. Ann Intern Med. 2016;165(7):473-81

10. United States Renal Data System. 2016 USRDS Annual Data Report: Epidemiology of Kidney Disease in the United States. Bethesda, MD: National Institute of Diabetes and Digestive and Kidney Diseases, National Institutes of Health; 2016.

11. Centers for Disease Control and Prevention (CDC). Vision Health Initiative (VHI). Common eye disorders. 2015. Available at: https://www. cdc.gov/visionhealth/basics/ced/index.html. Accessed July 5, 2018.

12. Rubin RR, Peyrot M. Quality of life and diabetes. Diabetes Metab Res Rev. 1999;15(3):205-18.

13. Luscombe FA. Health-related quality of life measurement in type 2 diabetes. Value Health. 2000;3 (Suppl 1):15-28.

14. Centers for Disease Control and Prevention (CDC). Self-rated fair or poor health among adults with diabetes-United States, 1996-2005. MMWR Morb Mortal Wkly Rep. 2006;55(45):1224-27.

15. Peyrot M, Rubin RR, Lauritzen T, Snoek FJ, Matthews DR, Skovlund SE. Psychosocial problems and barriers to improved diabetes management: results of the cross-national Diabetes Attitudes, Wishes and Needs (DAWN) Study. Diabet Med. 2005;22(10):1379-85.

16. Grandy S, Chapman RH, Fox KM; SHIELD Study Group. Quality of life and depression of people living with type 2 diabetes mellitus and those at low and high risk for type 2 diabetes: findings from the Study to Help Improve Early evaluation and management of risk factors Leading to Diabetes (SHIELD). Int J Clin Pract. 2008;62(4):562-68.

17. Grandy S, Fox KM; SHIELD Study Group. Change in health status (EQ5D) over 5 years among individuals with and without type 2 diabetes mellitus in the SHIELD longitudinal study. Health Qual Life Outcomes. 2012;10:99. 18. Hayes A, Arima H, Woodward M, et al. Changes in quality of life associated with complications of diabetes: results from the ADVANCE study. Value Health. 2016;19(1):36-41.

19. Schunk M, Reitmeir P, Rückert-Eheberg IM, et al. Longitudinal change in health-related quality of life in people with prevalent and incident type 2 diabetes compared to diabetes-free controls. PLoS One. 2017;12(5):e0176895. 20. Zhang P, Brown MB, Bilik D, Ackermann RT, Li R, Herman WH. Health utility scores for people with type 2 diabetes in U.S. managed care health plans: results from Translating Research Into Action for Diabetes (TRIAD). Diabetes Care. 2012;35(11):2250-56.

21. Gough SCL, Kragh N, Ploug UJ, Hammer M. Impact of obesity and type 2 diabetes on health-related quality of life in the general population in England. Diabetes Metab Syndr Obes. 2009;2:179-84.

22. Trikkalinou A, Papazafiropoulou AK, Melidonis A. Type 2 diabetes and quality of life. World J Diabetes. 2017;8(4):120-29.

23. Intensive blood-glucose control with sulphonylureas or insulin compared with conventional treatment and risk of complications in patients with type 2 diabetes (UKPDS 33). UK Prospective Diabetes Study (UKPDS) Group. Lancet. 1998;352(9131):837-53.

24. Quality of life in type 2 diabetic patients is affected by complications but not by intensive policies to improve blood glucose or blood pressure control (UKPDS 37). U.K. Prospective Diabetes Study Group. Diabetes Care. 1999;22(7):1125-36.

25. Alva M, Gray A, Mihaylova B, Clarke P. The effect of diabetes complications on health-related quality of life: the importance of longitudinal data to address patient heterogeneity. Health Econ. 2014;23(4):487-500.

26. Evans M, Khunti K, Mamdani M, et al. Health-related quality of life associated with daytime and nocturnal hypoglycaemic events: a time tradeoff survey in five countries. Health Qual Life Outcomes. 2013;11:90.

27. Simon D, de Pablos-Velasco P, Parhofer KG, et al. Hypoglycaemic episodes in patients with type 2 diabetes-risk factors and associations with patient-reported outcomes: The PANORAMA Study. Diabetes Metab. 2015;41(6):470-79. 
28. Polonsky WH, Fisher L, Hessler D, Edelman SV. Identifying the worries and concerns about hypoglycemia in adults with type 2 diabetes. J Diabetes Complications. 2015;29(8):1171-76.

29. Green AJ, Fox KM, Grandy S; SHIELD Study Group. Self-reported hypoglycemia and impact on quality of life and depression among adults with type 2 diabetes mellitus. Diabetes Res Clin Pract. 2012;96(3):313-18.

30. Lopez JM, Annunziata K, Bailey RA, Rupnow MF, Morisky DE. Impact of hypoglycemia on patients with type 2 diabetes mellitus and their quality of life, work productivity, and medication adherence. Patient Prefer Adherence. 2014;8:683-92

31. Alvarez-Guisasola F, Yin DD, Nocea G, Qiu Y, Mavros P. Association of hypoglycemic symptoms with patients' rating of their health-related quality of life state: a cross sectional study. Health Qual Life Outcomes. 2010;8:86.

32. Marrett E, Radican L, Davies MJ, Zhang Q. Assessment of severity and frequency of self-reported hypoglycemia on quality of life in patients with type 2 diabetes treated with oral antihyperglycemic agents: a survey study BMC Res Notes. 2011;4:251.

33. Ul-Haq Z, Mackay DF, Fenwick E, Pell JP. Meta-analysis of the association between body mass index and health-related quality of life among adults, assessed by the SF-36. Obesity. 2013;21(3):E322-27.

34. Wilding JPH. The importance of weight management in type 2 diabetes mellitus. Int J Clin Pract. 2014;68(6):682-91

35. Gonzalez JS, Fisher L, Polonsky WH. Depression in diabetes: have we been missing something important? Diabetes Care. 2011;34(1):236-39.

36. Rubin R. Diabetes and quality of life. Diabetes Spectrum. 2000;13:21-23.

37. Jain V, Shivkumar S, Gupta O. Health-related quality of life (hr-qol) in patients with type 2 diabetes mellitus. N Am J Med Sci. 2014;6(2):96-101.

38. Polonsky WH. Emotional and quality-of-life aspects of diabetes management. Curr Diab Rep. 2002;2(2):153-59.

39. American Diabetes Association. Economic costs of diabetes in the U.S. in 2012. Diabetes Care. 2013;36:1033-46.

40. Zhou Z, Zhang P, Hoerger P. Lifetime medical costs of treating type 2 diabetes and diabetic complications. Am J Prev Med. 2013;45(3):253-61.

41. Meng YY, Pickett MC, Babey SH, Davis AC, Goldstein H. Diabetes tied to a third of California hospital stays, driving health care costs higher. Policy Brief UCLA Cent Health Policy Res. 2014;(PB2014-3):1-7.

42. Costantino ME, Stacy JN, Song F, Xu Y, Bouchard JR. The burden of diabetes mellitus for Medicare beneficiaries. Popul Health Manag. 2014;17(5):272-78

43. Meyers JL, Parasuraman S, Bell KF, Graham JP, Candrilli SD. The highcost, type 2 diabetes mellitus patient: an analysis of managed care administrative data. Arch Public Health. 2014;72(1):6.

44. Saundankar V, Ellis J, Allen E, et al. Type 2 diabetes mellitus patients' healthcare costs related to inpatient hospitalizations: a retrospective administrative claims database study. Adv Ther. 2015;32(7):662-79.

45. Collins J, Abbass IM, Harvey R, et al. Predictors of all-cause 30 day readmission among Medicare patients with type 2 diabetes. Curr Med Res Opin. 2017;12:1-28.

46. Raval AD, Zhou S, Wei W, Bhattacharjee S, Miao R, Sambamoorthi U. 30-day readmission among elderly Medicare beneficiaries with type 2 diabetes. Popul Health Manag. 2015;18(4):256-64.

47. Fitch K, Pyenson BS, Iwasaki K. Medical claim cost impact of improved diabetes control for Medicare and commercially insured patients with type 2 diabetes. J Manag Care Pharm. 2013;19(8):609-20, 620a-20d. Available at: https://www.jmcp.org/doi/10.18553/jmcp.2013.19.8.609.

48. Hazel-Fernandez L, Li Y, Nero D, et al. Relationship of diabetes complications severity to healthcare utilization and costs among Medicare Advantage beneficiaries. Am J Manag Care. 2015;21(1):e62-70.

49. Candrilli SD, Meyers JL, Boye K, Bae JP. Health care resource utilization and costs during episodes of care for type 2 diabetes mellitus-related comorbidities. J Diabetes Complications. 2015;29(4):529-33.

50. Ward A, Alvarez P, Vo L, Martin S. Direct medical costs of complications of diabetes in the United States: estimates for event-year and annual state costs (USD 2012). J Med Econ. 2014;17(3):176-83.
51. Johnston SS, Sheehan JJ, Shah M, et al. Cardiovascular event costs in patients with type 2 diabetes mellitus. J Med Econ. 2015;18(12):1032-40. 52. Garber AJ, Abrahamson MJ, Barzilay JI, et al; American Association of Clinical Endocrinologists (AACE); American College of Endocrinology (ACE). Consensus statement by the American Association of Clinical Endocrinologists and American College of Endocrinology on the comprehensive type 2 diabetes management algorithm-2016 executive summary. Endocr Pract. 2016;22(1):84-113.

53. Dall TM, Yang W, Halder P, et al. Type 2 diabetes detection and management among insured adults. Popul Health Metr. 2016;14:43.

54. Lipska KJ, Yao X, Herrin J, et al. Trends in drug utilization, glycemic control, and rates of severe hypoglycemia, 2006-2013. Diabetes Care. 2017;40(4):468-75.

55. Shetty S, Secnik K, Oglesby AK. Relationship of glycemic control to total diabetes-related costs for managed care health plan members with type 2 diabetes. J Manag Care Pharm. 2005;11(7):559-64. Available at: https://www. jmcp.org/doi/10.18553/jmcp.2005.11.7.559.

56. Aagren M, Luo W. Association between glycemic control and shortterm healthcare costs among commercially insured diabetes patients in the United States. J Med Econ. 2011;14(1):108-14.

57. Foos V, Varol N, Curtis BH, et al. Economic impact of severe and nonsevere hypoglycemia in patients with type 1 and type 2 diabetes in the United States. J Med Econ. 2015;18(6):420-32.

58. Boulin M, Diaby V, Tannenbaum C. Preventing unnecessary costs of drug-induced hypoglycemia in older adults with type 2 diabetes in the United States and Canada. PLoS One. 2016;11(9):e0162951.

59. Campbell JA, Bishu KG, Walker RJ, Egede LE. Trends of medical expenditures and quality of life in U.S. adults with diabetes: the medical expenditure panel survey, 2002-2011. Health Qual Life Outcomes. 2017;15(1):70.

60. Valencia WM, Florez H. Pharmacological treatment of diabetes in older people. Diabetes Obes Metab. 2014;16(12):1192-203.

61. Dybicz SB, Thompson S, Molotsky S, Stuart B. Prevalence of diabetes and the burden of comorbid conditions among elderly nursing home residents. Am J Geriatr Pharmacother. 2011;9(4):212-23.

62. Huang ES. Management of diabetes mellitus in older people with comorbidities. BMJ. 2016;353:i2200.

63. Moghissi E. Management of type 2 diabetes mellitus in older patients: current and emerging treatment options. Diabetes Ther. 2013;4(2):239-56.

64. Kachroo S, Kawabata H, Colilla S, et al. Association between hypoglycemia and fall-related events in type 2 diabetes mellitus: analysis of a U.S. commercial database. J Manag Care Spec Pharm. 2015;21(3):243-53. Available at: https://www.jmcp.org/doi/10.18553/jmcp.2015.21.3.243.

65. Fu H, Curtis BH, Xie W, Festa A, Schuster DP, Kendall DM. Frequency and causes of hospitalization in older compared to younger adults with type 2 diabetes in the United States: a retrospective, claims-based analysis. J Diabetes Complications. 2014;28(4):477-81.

66. Munshi MN, Florez H, Huang ES, et al. Management of diabetes in longterm care and skilled nursing facilities: a position statement of the American Diabetes Association. Diabetes Care. 2016;39(2):308-18.

67. American Geriatrics Society Expert Panel on Care of Older Adults with Diabetes Mellitus, Moreno G, Mangione CM, Kimbro L, Vaisberg E. Guidelines abstracted from the American Geriatrics Society Guidelines for Improving the Care of Older Adults with Diabetes Mellitus: 2013 update. J Am Geriatr Soc. 2013;61(11):2020-26.

68. American Diabetes Association. Older adults. Sec. 10. In: Standards of medical care in diabetes 2016. Diabetes Care. 2016;39(Suppl 1):S81-85. 